\title{
Brief Communication: Premolar Enamel Formation: Completion of Figures for Aging LEH Defects in Permanent Dentition
}

Sarah A. Holt ${ }^{*}$, Donald J. Reid ${ }^{2}$, and Debbie Guatelli-Steinberg ${ }^{1}$

${ }_{1}^{1}$ Department of Anthropology, The Ohio State University, Columbus, OH 43210, USA

2Department of Oral Biology, School of Dental Sciences, University of Newcastle upon Tyne, Framlington Place, Newcastle upon Tyne NE2 4BW, UK

Key Words: Cross striations; Striae of Retzius; Enamel formation; Tooth growth; Dental development

ABSTRACT Variation in enamel formation has become increasingly important in comparative studies of dental development. Previously published work on the development of human enamel in groups from southern Africa and northern Europe has allowed for more accurate estimation of formation timing of linear enamel hypoplasias. Currently, although data for all

Variation in enamel formation provides many avenues of inquiry for those interested in comparisons of developmental life histories and aging enamel defects. For example, assessing the age of formation of linear enamel hypoplasias provides one line of evidence to estimate rates of childhood morbidity (e.g., Goodman and Song, 1998). Due to the nonlinear nature of enamel growth, linear regression formulas utilizing total crown height cannot be used to accurately estimate age of LEH formation (Reid and Dean, 2000; Martin et al., 2008; Ritzman et al., 2008). Data on population specific variation in crown formation times based on percentages of total growth are therefore of great use for accurately estimating LEH formation times.

The Reid and Dean (2006) methodology for histological growth assessment reflects the variability of enamel growth rates along the length of the crown and incorporates standard deviations reflecting inter-individual variation, avoiding the problem of applying linear statistical models to a nonlinear growth pattern. Studies completed using other methods, including dental radiographs of living children, suggested high levels of variation in crown formation time among human populations (e.g., Tompkins, 1996). The implication is that new research on LEH formation times conducted without the histological growth assessment method should draw on crown formation timing data specific to the population being studied (Reid and Dean, 2000; Reid et al., 2006; Martin et al., 2008). However, recent work in tooth types has been published, charts of enamel growth by decile useful in this type of estimation have been limited to molars and anterior teeth. This paper completes this series with a table and figure of mean formation times of human premolars for each decile of crown development using previously published histological data of daily enamel growth.

which incremental growth is assessed histologically has shown the anterior teeth and molars to be less variable between populations than previously reported (Reid and Dean, 2006).

Reid and Dean first published research comparing enamel formation times in populations from northern Europe (2000, 2006) and southern Africa (2006) that included tables presenting age in days for enamel formation at the completion of each decile of crown height for anterior teeth and molars (2006: 334-35). Figures depicting these data provided a visual guide for estimating $\mathrm{LEH}$ (2006: 343-44). These figures include an estimated age at mineralization based on previous histological studies (Reid et al., 1998; Reid and Dean, 2000; Antoine, 2001; Dean and Reid, 2001) but the authors note these initiation times are highly variable, as much as a full year in the M3 (Reid and Dean, 2006). A follow-up study (Reid et al., 2008) presented premolar data from these same populations, but did not provide charts of growth by decile of crown height or figures including initiation estimations.

\footnotetext{
${ }^{*}$ Correspondence to: Sarah Holt, Department of Anthropology, The Ohio State University 4034 Smith Laboratory. 174 W. 18th Ave. Columbus, Ohio 43210 USA

Email: holt.249@osu.edu

TEL: (614)330-6930
} 
TABLE 1. Age (in days) for enamel formation at each decile of crown height for molar teeth in each sample, $+/-1$ standard deviation

Southern African premolar tooth crown formation times

\begin{tabular}{|c|c|c|c|c|}
\hline & LP3 $n=33$ & $\mathrm{LP} 4 \mathrm{n}=28$ & UP3 n=45 & UP4 n=41 \\
\hline Initiation & 675 & 967 & 675 & 967 \\
\hline Cusp completion & $902+/-27$ & $1234+/-32$ & $910+/-36$ & $1211+/-42$ \\
\hline $10 \%$ complete & $943+/-29$ & $1270+/-34$ & $949+/-36$ & $1250+/-42$ \\
\hline $20 \%$ complete & $995+/-33$ & $1310+/-38$ & $993+/-34$ & $1292+/-42$ \\
\hline $30 \%$ complete & $1053+/-37$ & $1356+/-48$ & $1039+/-34$ & $1335+/-42$ \\
\hline $40 \%$ complete & $1116+/-48$ & $1408+/-53$ & $1092+/-37$ & $1382+/-46$ \\
\hline $50 \%$ complete & $1187+/-61$ & $1472+/-60$ & $1157+/-44$ & $1444+/-46$ \\
\hline $60 \%$ complete & $1266+/-75$ & $1548+/-74$ & $1239+/-52$ & $1526+/-50$ \\
\hline $70 \%$ complete & $1356+/-92$ & $1638+/-92$ & $1336+/-55$ & $1627+/-58$ \\
\hline $80 \%$ complete & $1452+/-109$ & $1741+/-110$ & $1443+/-62$ & $1737+/-70$ \\
\hline $90 \%$ complete & $1558+/-124$ & $1869+/-117$ & $1570+/-71$ & $1854+/-77$ \\
\hline Crown completion & $1665+/-141$ & $1986+/-124$ & $1703+/-76$ & $1974+/-82$ \\
\hline \multicolumn{5}{|c|}{ Northern European Crown Formation Times } \\
\hline & LP3 $n=33$ & $\mathrm{LP} 4 \mathrm{n}=22$ & UP3 n=34 & $\mathrm{UP} 4 \mathrm{n}=44$ \\
\hline Initiation & 675 & 967 & 675 & 967 \\
\hline Cusp completion & $891+/-44$ & $1231+/-36$ & $952+/-65$ & $1225+/-63$ \\
\hline $10 \%$ complete & $952+/-54$ & $1276+/-41$ & $1010+/-61$ & $1284+/-52$ \\
\hline $20 \%$ complete & $1018+/-68$ & $1328+/-43$ & $1068+/-52$ & $1338+/-48$ \\
\hline $30 \%$ complete & $1088+/-80$ & $1381+/-52$ & $1132+/-50$ & $1390+/-44$ \\
\hline $40 \%$ complete & $1164+/-96$ & $1441+/-65$ & $1203+/-49$ & $1448+/-48$ \\
\hline $50 \%$ complete & $1256+/-113$ & $1509+/-76$ & $1285+/-53$ & $1521+/-52$ \\
\hline $60 \%$ complete & $1359+/-135$ & $1594+/-84$ & $1389+/-60$ & $1613+/-62$ \\
\hline $70 \%$ complete & $1481+/-164$ & $1697+/-98$ & $1518+/-66$ & $1724+/-71$ \\
\hline $80 \%$ complete & $1614+/-194$ & $1817+/-122$ & $1663+/-78$ & $1856+/-85$ \\
\hline $90 \%$ complete & $1766+/-224$ & $1948+/-143$ & $1838+/-84$ & $1998+/-98$ \\
\hline Crown completion & $1908+/-253$ & $2071+/-162$ & $2011+/-92$ & $2134+/-110$ \\
\hline
\end{tabular}

Currently, therefore, although enamel growth patterns of these populations have been established, the series of charts and figures providing a visual guide for estimating population specific LEH formation times has lacked the published information on premolars. This communication completes the publication of this series of figures by presenting premolar enamel growth by decile for populations from southern Africa and northern Europe (Table 1, Fig. 1).

\section{MATERIALS AND METHODS}

Data from 147 premolars collected from two populations, southern Africa and Newcastle, England, (northern Europe), were used to create tables of enamel formation (Reid et al., 2008). The premolars were originally collected after extraction during oral surgery and histological thin sections were prepared for polarized light microscopy (Reid and Dean, 2006). Individual periodicity for each tooth was established by counting daily cross-striations in enamel, and formation times for each decile of crown height was then recorded using measurements of long-period striations corresponding to the perikymata on the external crown surface. Initiation ages of crown mineralization for both samples were estimated from a third, French sample (Reid et al., 1998). By adding these decile data to age at initiation of crown mineralization, formation times for both cuspal and lateral enamel formation in days was determined (see Reid et al., 2008 and Reid and Dean, 1998 for full discussion of methodology). 


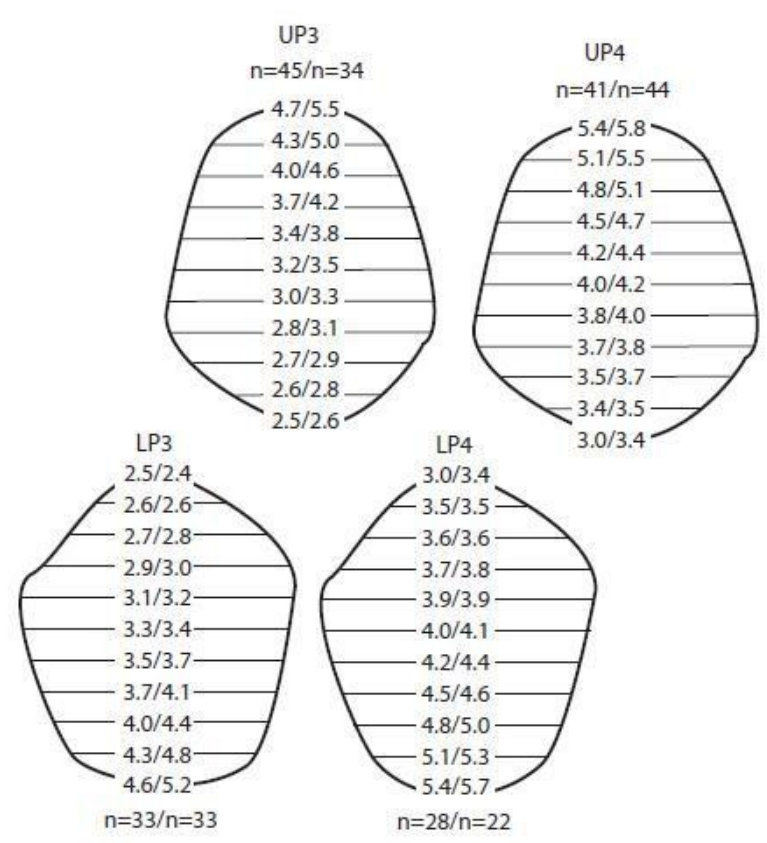

Fig. 1. Mean estimates for the chronological ages of enamel formation in premolars for each decile of crown length rounded up or down to 0.1 year for the southern African sample vs. the northern European sample. Both initiation and cuspal enamel formation are included in these estimates.

Although extremely preliminary research by one of the authors on mineralization initiation times specific to these populations supports the current expectation that mineralization timing will vary, conclusive data from large scale studies remains unavailable. Given the absence of data, the significance of future publications in this area on the chart presented here would be speculative, however, the authors intend to update the available charts as new data are available.

\section{DISCUSSION}

Although total crown formation time was found to be significantly different between the populations $(\mathrm{p}<0.00)$ (see Reid et al., 2008 for full discussion of statistical methods), as with the anterior and molar teeth, premolar formation time between populations is more similar than radiographic studies once suggested (e.g., Tompkins, 1996). While the southern African sample crowns formed consistently more quickly, the means of both samples range only from 0.3 years difference in the lower P4 to 0.8 years difference in the upper P3. Thus, the small amount of variation may not be particularly meaningful in comparisons of human populations (Reid and Dean, 2006; Martin

et al., 2008).

Because this method presents formation time by decile, the estimation of age during LEH formation can be independent from any variation in length of the crown. This more accurate method has already been widely used for the anterior teeth and molars (e.g., Reid and Dean, 2000; 2006). The addition of premolar data will allow for comparisons of LEH within the dentition of individuals, specifically to match LEH manifestations of a single stress event across premolars and other teeth within an individual. Despite the fact that enamel growth is not linear, Martin et al. (2008) found no difference between a linear and nonlinear interpolation for ages that fall between the established deciles. They conclude that a linear interpolation is sufficient to age defects that fall within a decile. While we await further histological data from other populations, the charts presented here (Table 1, Fig. 1) make it possible to provide estimates of LEH formation times that utilize the population specific information currently available from two geographically distant populations.

\section{CONCLUSION}

Recent studies applying histological methods to establish enamel formation timing have shown less variation in modern human premolar formation between populations than previous methodologies; however, statistically significant differences have been documented between a large sample of northern European and southern African individuals (Reid and Dean, 1998; Reid et al., 2008). Previously published data on histological timing of each decile of premolar tooth crowns can be used to estimate timing of LEH formation without destructive histological sectioning. This paper presents a summary of the previously published data on crown formation variation and presents a graphic diagram of the premolar formation times by decile drawn from histological analysis (Table 1, Fig. 1). In addition to providing previously unpublished data on the mineralization initiation estimates used to create these decile formation charts, it is the hope of the authors that including a visual representation of premolar crown formation by decile to the existing charts for other tooth types will allow for more practical application of the known formation timing to analysis of the external enamel of premolars. 


\section{LITERATURE CITED}

Antoine, D. 2001. Evaluating the periodicity of incremental structures in dental enamel as a means of studying growth in children from past populations. Ph.D. Dissertation, University of London.

Dean, MC, Reid, DJ. 2001. Perikymata spacing and distribution on hominid anterior teeth. Am J Phys Anthropol 116:209-215.

Goodman AH, Song RJ. 1998. Sources of variation in estimated ages at formation of linear enamel hypoplasias. In: Hoppa RD, FitzGerald CM, editors. Human growth in the past: Studies from bones and teeth. Cambridge: Cambridge University Press 210-240.

Martin SA, Guatelli-Steinberg D, Sciulli PW, Walker PL. 2008. Brief Communication:

Comparison of Methods for Estimating Chronological Age at Linear Enamel Formation on Anterior Dentition. Am J Phys Anthropol 135:362-265.

Reid DJ, Beynon AD, Rameriez Rozzi FV. 1998. Histological reconstruction of dental development in four individuals from a medieval site in Picardie, France. J Hum Evol 35:463-477.

Reid DJ, Dean MC. 2000. Brief communication: the timing of linear hypoplasias on human anterior teeth. Am J Phys Anthropol 113:135-139.

Reid DJ, Dean MC. 2006. Variation in modern human enamel formation times. J Hum Evol 50:329-346.

Reid DJ, Guatelli-Steinberg D, Walton P. 2008. Variation in modern human premolar enamel formation times: Implications for Neandertals. J Hum Evol 54:225-235.

Ritzman TB, Baker BJ, Schwartz GT. 2008. A Fine Line: A Comparison of Methods for Estimating Ages of Linear Enamel Hypoplasia Formation. Am J Phys Anthropol 135:348-361.

Tompkins, RL, 1996. Human population variability in relative dental development. Am J Phys Anthropol 99:79-102. 